\title{
Assistência de enfermagem aos pacientes portadores de alzheimer: uma revisão integrativa
}

RESUMO | O Alzheimer se caracteriza como uma demência neurodegenerativa, fatal e incurável, acometendo, principalmente os idosos. Objetivo: Evidenciar o estado da arte acerca da assistência de enfermagem ao portador de Alzheimer. Método: Tratase de uma revisão integrativa da literatura acerca da Assistência de Enfermagem ao portador de Alzheimer, nos anos de 2016 a 2020, dentro das seguintes bases de dados: SciELO e LILACS, utilizando como estratégia de busca os descritores combinados com operadores booleanos "enfermagem" and "Alzheimer". Resultados: A amostra do estudo foi composta por sete artigos, evidenciando o papel do enfermeiro, junto ao cuidador e com o portador da Doença de Alzheimer, além de mostrar necessárias ações de promoção da saúde e prevenção de agravos. Conclusão: A doença de Alzheimer se configura como um desafio para a sociedade contemporânea, sendo que a Enfermagem tem papel fundamental no cuidado ao portador e ao cuidador.

Palavras-chaves: Doença de Alzheimer; Enfermagem; Cuidados de Enfermagem; Idoso Fragilizado.

\begin{abstract}
Alzheimer's is characterized as a neurodegenerative, fatal and incurable dementia, affecting mainly the elderly. Objective: To highlight the state of the art about nursing care for Alzheimer's patients. Method: This is an integrative review of the literature on Nursing Care for Alzheimer's patients, in the years 2016 to 2020, within the following databases: SciELO and LILACS, using as search strategy the descriptors combined with Boolean operators "nursing "And" Alzheimer ". Results: The study sample consisted of seven articles, evidencing the role of the nurse, with the caregiver and with the Alzheimer's Disease patient, in addition to showing necessary actions for health promotion and disease prevention. Conclusion: Alzheimer's disease is a challenge for contemporary society, with Nursing playing a fundamental role in caring for patients and caregivers.
\end{abstract}

Keywords: Alzheimer Disease; Nursing; Nursing Care; Frail Elderly.

RESUMEN I El Alzheimer se caracteriza por ser una demencia neurodegenerativa, fatal e incurable, que afecta principalmente a los ancianos. Objetivo: Destacar el estado del arte en la atención de enfermería a los pacientes con Alzheimer. Método: Se trata de una revisión integradora de la literatura sobre Atención de Enfermería para pacientes con Alzheimer, en los años 2016 a 2020 , dentro de las siguientes bases de datos: SciELO y LILACS, utilizando como estrategia de búsqueda los descriptores combinados con operadores booleanos "enfermería "Y" Alzheimer ". Resultados: La muestra de estudio estuvo conformada por siete artículos, evidenciando el rol de la enfermera, con el cuidador y con el paciente con Enfermedad de Alzheimer, además de mostrar las acciones necesarias para la promoción de la salud y prevención de la enfermedad. Conclusión: la enfermedad de Alzheimer es un desafío para la sociedad contemporánea, siendo la Enfermería un papel fundamental en el cuidado de pacientes y cuidadores.

Palabras claves: Enfermedad de Alzheimer; Enfermería; Atención de Enfermería; Anciano Frágil.

\section{Sabrina Piccineli Zanchettin Silva}

Enfermeira. Professora, Graduação em Enfermagem, Centro universitário de Lins - UNILINS. Lins (SP), Brasil. Mestre em biologia e envelhecimento pela Faculdade de Medicina de Marilia - FAMEMA.

ORCID: 0000-0002- 5763-6814

\section{Allison Vinicius Bernardo}

Discente da Graduação em Enfermagem, Centro Universitário de Lins - UNILINS. Lins (SP), Brasil.

ORCID: 0000-0002-5636-3711

\section{Caroline Lima Noronha Lô}

Discente da Graduação em Enfermagem, Centro Universitário de Lins - UNILINS. Lins (SP), Brasil.

ORCID: 0000-0002-9408-7420

\section{Giovana Verdeli Teodoro Campeiro}

Discente da Graduação em Enfermagem, Centro Universitário de Lins - UNILINS. Lins (SP), Brasil.

ORCID: 0000-0002-1245-948x

\section{Lucas Rocha dos Santos}

Discente da Graduação em Enfermagem, Centro Universitário de Lins - UNILINS. Lins (SP), Brasil.

ORCID: 0000-0002-5943-2911

\section{INTRODUÇÃO}

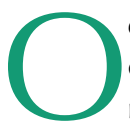
envelhecimento é um processo inevitável, ocorrendo mudanças fisiológicas, psicológicas e morfológicas ${ }^{(1)}$. Dados epidemiológicos evidenciam crescimento dos idosos justificado, dentre outras razões, pela queda da natalidade.

As estimativas projetam que em 2025, haverá 1,2 bilhões de idosos no mundo, e no Brasil, serão 32 milhões, deixando o Brasil em sexto lugar no ranking mundial de pessoas idosas (acima dos 60 anos). Perante a esse contexto, as doenças crônico-degenerativas, assim como as demências, muito prevalentes nos idosos, constituem-se como uma preocupação para a área da saúde ${ }^{(2)}$.

A Doença de Alzheimer ${ }^{(3)}$ se caracteriza como uma demência neurodegenerativa, fatal e incurável. A doença é causada pela intoxicação e morte dos neurônios, e se manifesta através da perda de funções cognitivas (memória, orientação, atenção e linguagem). A Doença de Alzheimer é uma doença que tratável, e quanto mais rápido seu diagnóstico, mais seu avanço é retardado, gerando uma melhor qualidade de vida ao portador da doença, e à família. Há uma estimativa mundial de que existam cerca de 35,6 miIhões de pessoas com a DA. No Brasil, há 
Zanchettin Silva, S.P.; Bernado, A.V.; Lô, C.L.N.; Campeiro, G.V.T.; Santos, L.R.

cerca de 1,2 milhão de portadores, sendo sem diagnóstico a maioria destes $\operatorname{casos}^{(4)}$. Sabe-se que a Doença de Alzheimer acomete a maioria das pessoas na idade avançada, geralmente este cliente não possui qualidade de vida, sendo este um momento onde aparece com maior frequência os problemas de saúde, com isso, percebe-se a importância da atuação dos cuidadores, pois os portadores da doença, necessitam de ajuda constantemente para realizar atividades rotineiras ${ }^{(3)}$.

$\mathrm{Na}$ fase inicial, os portadores de Alzheimer podem apresentar um perfil de veIhice, sendo isso um grande problema, pois a maioria são ignorados, fazendo com que não seja detectado a doença nesta fase, na fase tardia o portador apresenta um perfil de total dependência, os distúrbios nessa fase são graves, e os sinais e sintomas físicos são evidentes, apresentando perda de funções cognitivas, perda da capacidade intelectual, nessa fase o cliente pode até perder as habilidades que possuía antes de se adoecer, podendo apresentar dificuldade para andar e ter um perfil de agressivo ${ }^{(5)}$.

Como trata-se de uma doença neurodegenerativa, os problemas aparecem gradativamente, e com maior frequência, com isso aumentando cada vez mais o trabalho não somente do cuidador, e sim da enfermagem, familiares, e dentre outros profissionais pelo tempo integral, mesmo fora do ambiente dos centros de referência; hospitais e clínicas, podem encarregar-se de detalhes relativos à alimentação, ambiente e outros aspectos que podem elevar a qualidade de vida dos pacientes ${ }^{(3)}$.

Assim, a atuação do enfermeiro, na prestação da assistência da enfermagem, seja através da realização de orientações, apoio familiar, execução de técnicas, contribuíram para melhoria da qualidade de vida do portador ${ }^{(6)}$.

Perante ao exposto o presente estudo tem como objetivo evidenciar o estado da arte acerca da assistência de enfermagem ao portador de Alzheimer.

\section{MÉTODO}

Trata-se de uma revisão integrativa ${ }^{(7)}$, com a seguinte pergunta delineadora: Como se dá a assistência de enfermagem aos idosos portadores de Alzheimer?

A seleção da amostra deu-se por meio do acesso às bases de dados: Literatura Latino Americana e do Caribe em Ciências da Saúde (LILACS) e SciELO, sem determinar um campo específico de busca (article title; abstract; keywords...), mas optando por all fields. Utilizaram-se como estratégia de busca os descritores controlados combinados com operadores booleanos "enfermagem" and "Alzheimer".

Adotaram-se como critérios de inclusão: artigos completos com resumos e relacionados ao objeto de pesquisa, com restrição de idiomas (português), indexados nas bases de dados referidas, no período de 2016 a 2020. A coleta de dados ocorreu entre os meses de agosto e setembro de 2020.

Foram selecionados seis artigos do SciELO e 1 artigo da LILACS, resultando em uma amostra final de sete artigos (Figura 1).

Após a seleção dos artigos, realizou-se leitura criteriosa do título e do resumo; em seguida, foram excluídos aqueles que não respondiam à pergunta norteadora e que se repetiam entre as bases de dados. Para a análise e posterior síntese dos artigos que atenderam aos critérios de inclusão, elaborou-se um instrumento para coleta de dados que contemplasse dados referentes à publicação, à base de dados, aos objetivos e às principais conclusões.

\section{RESULTADOS}

A amostra final desta revisão foi constituída por sete artigos, selecionados pelos critérios de inclusão previamente estabelecidos. Destes, doze foram encontrados na base de dados SciELO e um na LILACS, como pode ser verificado no quadro 1.

\section{DISCUSSÃO}

Ao falar-se em assistência de enfermagem, fala-se em Sistematização da Assistência de Enfermagem (SAE). A taxonomia NANDA possibilita aos enfermeiros identificar os principais diagnósticos dos portadores de DA, destacando-se entre eles: nutrição, mobilidade física, autocuidado, memória e comunicação prejudicadas,
Figura 1 - Fluxograma de constituição da amostra, 2020.
Amostra Final
SCIELO : 1
LILACS: 6
TOTAL: 7 

periódico, autores, título do artigo e principais resultados - Lins, 2020.

\begin{tabular}{|c|c|c|c|}
\hline Base de dados/ano & Periódico/ Autores & Título do artigo & Principais resultados \\
\hline LILACS/ 2019 & $\begin{array}{l}\text { Rev Rene (Online)/ Barbosa, Maria } \\
\text { Emília Marcondes; Bertelli, Ellen } \\
\text { Vanuza Martins; Scolari, Giovana } \\
\text { Aparecida de Souza; Bortolanza, } \\
\text { Marciane Conti Zornita; Higarashi, } \\
\text { leda Harumi; Carreira, Lígia. }\end{array}$ & $\begin{array}{l}\text { Vulnerabilidade clínica e } \\
\text { funcional de idosos cuidado- } \\
\text { res de idosos com doença de } \\
\text { Alzheimer }\end{array}$ & $\begin{array}{l}\text { Identificou-se maior risco de vulnerabilidade relacio- } \\
\text { nada à autopercepção de saúde, cognição e humor. } \\
\text { Funcionalidade preservada nas funções executivas, } \\
\text { comunicação, mobilidade e comorbidades múlti- } \\
\text { plas. Conclusão a maioria dos idosos cuidadores } \\
\text { apresentou risco de fragilização, porém realizam o } \\
\text { cuidado ao familiar com Alzheimer. Profissionais e } \\
\text { instituições devem atuar na prevenção do declínio } \\
\text { funcional desses cuidadores idosos. }\end{array}$ \\
\hline LILACS/ 2017 & $\begin{array}{l}\text { Texto \& contexto enferm/ Ilha, Silo- } \\
\text { mar; Santos, Silvana Sidney Costa; } \\
\text { Backes, Dirce Stein; Barros, Edaiane } \\
\text { Joana Lima; Pelzer, Marlene Teda; } \\
\text { Gautério-Abreu, Daiane Porto. }\end{array}$ & $\begin{array}{l}\text { Gerontotecnologias utilizadas } \\
\text { pelos familiares/cuidadores de } \\
\text { idosos com alzheimer: contri- } \\
\text { buição ao cuidado complexo }\end{array}$ & $\begin{array}{l}\text { Os familiares/cuidadores de pessoas idosas com } \\
\text { doença de Alzheimer vivenciam dificuldades nos } \\
\text { aspectos físico, mental e social, para as quais desen- } \\
\text { volvem/empregam gerontotecnologias na forma de } \\
\text { produto e de processo/conhecimento/estratégias, } \\
\text { para auxiliá-los no cuidado/convivência com a } \\
\text { pessoa idosa com a doença de Alzheimer. }\end{array}$ \\
\hline LILACS/ 2017 & $\begin{array}{l}\text { Rev. Pesqui. (Univ. Fed. Estado Rio J., } \\
\text { Online)/ Souza, Ítala Paris de; Araújo, } \\
\text { Laura Filomena Santos de; Bellato, } \\
\text { Roseney. }\end{array}$ & $\begin{array}{l}\text { A dádiva e o cuidado no } \\
\text { tempo vivido em família / } \\
\text { La dádiva y el cuidado en el } \\
\text { tiempo del vivido en familia }\end{array}$ & $\begin{array}{c}\text { Mostrou a importância da atuação dos profissionais } \\
\text { de saúde na produção de boas práticas, como forma } \\
\text { de apoio à família. }\end{array}$ \\
\hline LILACS/ 2017 & $\begin{array}{l}\text { Rev Rene (Online)/ Sales, Marta } \\
\text { Gabriele Santos; Menezes, Tânia } \\
\text { Maria de Oliva }\end{array}$ & $\begin{array}{c}\text { Significado da sexualidade } \\
\text { para o cônjuge cuidador de } \\
\text { pessoa idosa com doença de } \\
\text { Alzheimer }\end{array}$ & $\begin{array}{l}\text { Revelou-se falta de conhecimento acerca do termo } \\
\text { "sexualidade", bem como a vergonha de falar sobre } \\
\text { a temática. A sexualidade esteve presente na vida } \\
\text { de alguns e foi abolida da vida de outros. Identificou } \\
\text { que a presença da doença repercute na vida e sexu- } \\
\text { alidade do portador e sua relação conjugal. }\end{array}$ \\
\hline LILACS/ 2017 & $\begin{array}{l}\text { Ciênc. cuid. Saúde/ Faria, Érica Bea- } \\
\text { triz Alves; Scardoelli, Marcia Glaciela } \\
\text { da Cruz; Castro, Vivian Carla de; } \\
\text { Nishida, Fernanda Shizue. }\end{array}$ & $\begin{array}{l}\text { Vivências de Cuidadores } \\
\text { Familiares de Pessoas Idosas } \\
\text { com Doença de Alzheimer }\end{array}$ & $\begin{array}{l}\text { Foi constatado que a vivência do processo de } \\
\text { adoecimento gera sofrimento, tristeza e insegu- } \\
\text { rança, trazendo grandes mudanças no contexto } \\
\text { familiar. } 0 \text { enfermeiro, além da assistência ao } \\
\text { idoso com DA, pode oportunizar diálogos e defi- } \\
\text { nição conjunta de estratégias de cuidados para a } \\
\text { convivência com a doença. }\end{array}$ \\
\hline LILACS/ 2015 & $\begin{array}{l}\text { Espaç. saúde (Online)/ Pizolotto, } \\
\text { Ana Laura Zuchetto; Leite, Marinês } \\
\text { Tambara Leite; Hildebrandt, Leila Ma- } \\
\text { riza; Costa, Marta Cocco da; Resta, } \\
\text { Darielli Gindri } \\
\end{array}$ & $\begin{array}{l}\text { Organização da família no } \\
\text { cuidado ao idoso com doença } \\
\text { de Alzheimer }\end{array}$ & $\begin{array}{l}\text { Observa-se a importância do vínculo entre os } \\
\text { familiares e o idoso com doença de Alzheimer. É } \\
\text { relevante a atenção da equipe de enfermagem } \\
\text { prestando assistência necessária. }\end{array}$ \\
\hline LILACS/ 2015 & $\begin{array}{l}\text { Rev. bras. Enferm/ Cruz, Thiara } \\
\text { Joanna Peçanha da; Sá, Selma Petra } \\
\text { Chaves; Lindolpho, Mirian da Costa; } \\
\text { Caldas, Célia Pereira. }\end{array}$ & $\begin{array}{l}\text { Estimulação cognitiva para } \\
\text { idoso com Doença de Alzhei- } \\
\text { mer realizada pelo cuidador }\end{array}$ & $\begin{array}{l}\text { A realização da estimulação cognitiva leve, reali- } \\
\text { zada pelo cuidador, após orientação do enfermeiro } \\
\text { tornou-o menos ansioso e promoveu maior compre- } \\
\text { ensão da doença. }\end{array}$ \\
\hline
\end{tabular}

confusão crônica, baixa autoestima crônica, ansiedade, dentre outros ${ }^{(8)}$.

Já as intervenções podem incluir: auxílio na alimentação e designação de horários para o idoso, auxílio na deambulação, oferecendo comandos precisos, auxiliar na higiene pessoal, dialogar estimulando a pessoa a lembrar-se de sua vida, auxiliar em jogos que ajudem a memória ficar ativa, ajudar na autoestima estimulando a pessoa a se arrumar e se vestir conforme o gosto, lembrá-la de que está sempre boni- ta, auxiliar e comunicar a família sobre o estado do doente, ajudar na melhora e nas conversas entre o doente e as pessoas que se encontram ao seu redor ${ }^{(8)}$.

Apontam a Teoria Transcultural de Madeleine Leininger, como um modelo para 
o cuidado da enfermagem ao portador do Mal de Alzheimer. Nessa teoria, o enfermeiro atua assistindo o portador, a cuidador, a família, e a comunidade, além de apoiar o portador, seja qual for a cultura que ele está inserido, considerando o seu contexto, valores, crenças, estilo de vida e práticas do próprio indivíduo e familiar, sempre orientando a evitar mudanças ${ }^{(9)}$.

Correia et al. ${ }^{(10)}$ classifica os cuidados de enfermagem em nove itens: "Atividades Essenciais de Vidas Diárias", como o auxílio no banho e nos cuidados gerais, cuidando para que não haja quedas. "Alimentação", uma vez que os portadores não possuem boa aceitação, onde o enfermeiro incentiva o preparo da sua própria refeição. "Melhora do sono", uma vez que o idoso portador possui dificuldades para dormir, e o enfermeiro age aplicando a musicoterapia e a atividade física em seu cotidiano. "Arteterapia", que ajuda a curar os anseios psicológicos, cria momentos de autorreflexão, compreensão e empatia, e melhora os parâmetros fisiológicos.

"Músicoterapia", que com o auxílio do enfermeiro, ajuda estimular a memória, funções cognitivas, e influencia positivamente nos aspectos da vida. "Aumento da socialização", que ajuda o idoso participar de atividades em família e em sociedade. "Administração de medicamentos", onde o enfermeiro tem o dever de orientar quanto a administração dos fármacos de forma assertiva, e sem atrasos. "Estimulação Cognitiva" onde foram adotadas as estratégias de musicoterapia, estimulação de memória e oficinas para o estímulo das funções cognitivas. "Controle da dor", onde novamente a musicoterapia foi adotada ${ }^{(10)}$.

O enfermeiro tem papel importante, junto ao cuidador e com a pessoa idosa que possui DA. Ele precisa desenvolver ações de promoção da saúde e prevenção de agravos para ambos, cuidador e pessoa que é cuidada, com o objetivo de que tenham uma vida mais saudável e com qualidade. Além disso, pode atuar junto à equipe de saúde vinculada às unidades básicas de saúde, orientando-a e qualificando-a, para que possa ofertar suporte ao familiar e ao paciente ${ }^{(11)}$.

É importante que o enfermeiro habilidosamente domine técnicas de avaliação e verifique se mudanças específicas de comportamento da pessoa com Doença de Alzheimer estão trazendo dor, sofrimento para o cuidador, com trabalho subsequente para identificar, classificar e avaliar estratégias em uso. A seguir, vem a demanda de aprender, personalizar e ensinar técnicas alternativas de intervenção e manejo baseadas nas necessidades de cuidado do binômio, trazidas pelo cuidador ${ }^{(12)}$.
Em Instituições de Longa Permanência, constatou-se que o cuidado assistencial referente à higiene, dieta, lazer, repouso, dentre outros, se encontra satisfatório. Ademais, deve-se considerar os limites de cada idoso, sendo promovida uma assistência efetiva, com benefícios que não só servem para o corpo, como também para a mente. Observou-se, também, o respeito por parte dos profissionais quando o idoso recusa a assistência, sendo sua vontade respeitada ${ }^{(13)}$.

Para uma assistência adequada que atenda às necessidades e preserve a integridade dos idosos são necessários aceitação, informação e flexibilidade por parte dos cuidadores, para que haja uma satisfatória adaptação à nova condição do internado(13).

\section{CONCLUSÃO}

Considerando o envelhecimento populacional e a significância da doença de Alzheimer entre eles, assim como suas repercussões na saúde, ela se configura como um desafio para a sociedade contemporânea. Dentro desse cenário, o presente estudo identificou-se a relevância da atuação da enfermagem no cuidado ao portador, assim como, para o cuidador frente a Doença de Alzheimer, sendo fundamental o conhecimento acerca do manejo da doença.

\section{Referências}

1. Lima, Emanuelle et al. A sistematização da assistência de Enfermagem e o mal de alzheimer, ReBIS Revista Brasileira Interdisciplinar de Saúde Volume 10 - n. 1 - Viçosa-MG - JAN/DEZ 2018. [citado 30 de junho de 2020]

2. Guimarães, T. M. R. et al. Assistência de enfermagem aos pacientes com Doença de Alzheimer em cuidados paliativos: revisão sistemática. Revista Eletrônica Acervo Saúde. Vol.Sup.n.38. Recife-PE, Janeiro de 2020. [citado 30 de junho de 2020]

3. Ministério da Saúde. Alzheimer: o que é, causas, sintomas, tratamento, diagnóstico e prevenção. Disponível em: <https://saude.gov.br/saude-de-a-z/ alzheimer> Acesso em: 07 de abril de 2020.

4. Abraz, Associação Brasileira de Alzheimer. 0 que é Alzheimer. Disponível em <http://abraz.org.br/web/sobre-alzheimer/o-que-e-alzheimer/> Acesso em 07 de abril de 2020.

5. Fef, Fundação educacional de Fernandópolis. Perfil do portadores da doença de alzheimer de comunidades da cidade de fernandópolis, 2020. [citado 30 de junho de 2020]

6. Faria KA, OLENIRA C. et al. Atuação da enfermagem frente ao paciente com doença de alzheimer. volume I, $\mathrm{n}^{\circ} \mathrm{I}$, Junho/2018. [citado 30 de junho de 2020] 7. Sousa LMM, Marques-Viera CMA, Severino SSP, Antunes AV. Metodologia de Revisão Integrativa da Literatura em Enfermagem. Revista Investigação Enfermagem [Internet]. 2017 [cited 2020 Apr 17];2:17-26. Available from: https://
www.researchgate.net/publication/321319742_Metodologia_de_Revisao_Integrativa_da_Literatura_em_Enfermagem.

8. Farfan AEO, Farias GB, Rohrs RMS, Magalhães MSSP, Silva DF, Schulz RS. Cuidados de enfermagem a pessoas com demência de alzheimer. Rev. Cuid art. São Paulo: Catanduva, 2017. [citado 30 de junho de 2020], 11(1):138-145. 9. Barbosa MEM, Corso ER, Scolari GAS, Carreira L. Interdisciplinaridade do cuidado a idosos com doença de Alzheimer: reflexão à luz das teorias de Leininger e de Heller. Escola Anna Nery 24(1) 2020. [citado dia 28 de junho de 2020]. 10. Correa LP, Braga TR, Malaquias LC, Bessa MEP, Marques MB. Intervenções de enfermagem nos cuidados aos pacientes idosos com Alzheimer: revisão integrativa. Rev Enferm UFPI. 2016 Jan-Mar;5(1):84-88. [citado dia 28 de junho de 2020].

11. Pizzoloto ALZ, Leite MT, Hildebrandt LM, Costa MCC, Resta DG. Organização da família no cuidado ao idoso com doença de Alzheimer. Rev. Esp. p. a Saúd. Londrina. 2015. [citado dia 30 de junho de 2020], 16(4): 41-54.

12. Marins AMF, Hansel CG, Silva J. Mudanças de comportamento em idosos com Doença de Alzheimer e sobrecarga para o cuidador. Esc Anna Nery. [internet]. 2016. [citado em 30 de junho de 2020], 20(2):352-356.

13. Fernandes MA, Sousa JWOG, Sousa WS de et al. Cuidados prestados ao idoso com alzheimer em instituições de longa permanência. Rev enferm UFPE on line., Recife, 12(5):1346-54, maio., 2018 [citado em 30 de junho de 2020], 